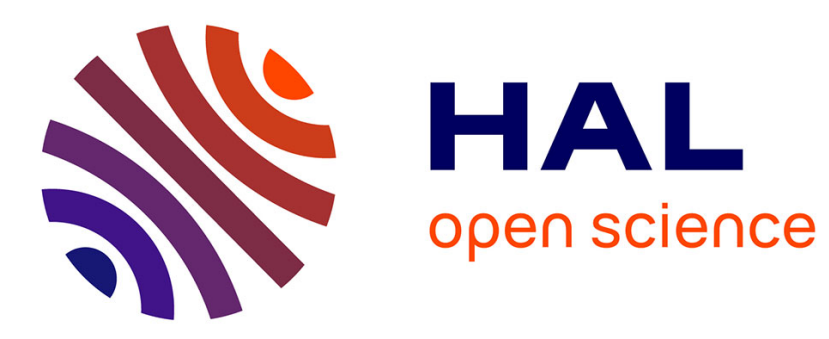

\title{
The effect of wall slip on the stability of the Rayleigh-Bénard Poiseuille flow of viscoplastic fluids
}

Christel Métivier, Albert Magnin

\section{To cite this version:}

Christel Métivier, Albert Magnin. The effect of wall slip on the stability of the Rayleigh-Bénard Poiseuille flow of viscoplastic fluids. Journal of Non-Newtonian Fluid Mechanics, 2011, 166 (14-15), pp.839-846. 10.1016/j.jnnfm.2011.04.017 . hal-01665072

\section{HAL Id: hal-01665072 \\ https://hal.univ-lorraine.fr/hal-01665072}

Submitted on 19 Dec 2017

HAL is a multi-disciplinary open access archive for the deposit and dissemination of scientific research documents, whether they are published or not. The documents may come from teaching and research institutions in France or abroad, or from public or private research centers.
L'archive ouverte pluridisciplinaire HAL, est destinée au dépôt et à la diffusion de documents scientifiques de niveau recherche, publiés ou non, émanant des établissements d'enseignement et de recherche français ou étrangers, des laboratoires publics ou privés. 


\section{Accepted Manuscript}

The effect of wall slip on the stability of the Rayleigh-Bénard Poiseuille flow of viscoplastic fluids

Christel Métivier, Albert Magnin

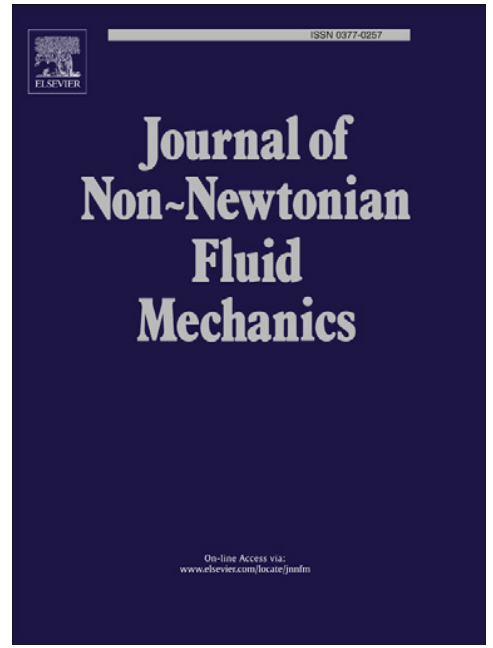

PII: S0377-0257(11)00115-7

DOI: 10.1016/j.jnnfm.2011.04.017

Reference: JNNFM 3228

To appear in: Journal of Non-Newtonian Fluid Mechanics

Received Date: $\quad 8$ February 2011

Revised Date: $\quad 19$ April 2011

Accepted Date: $\quad 21$ April 2011

Please cite this article as: C. Métivier, A. Magnin, The effect of wall slip on the stability of the Rayleigh-Bénard Poiseuille flow of viscoplastic fluids, Journal of Non-Newtonian Fluid Mechanics (2011), doi: 10.1016/j.jnnfm. 2011.04.017

This is a PDF file of an unedited manuscript that has been accepted for publication. As a service to our customers we are providing this early version of the manuscript. The manuscript will undergo copyediting, typesetting, and review of the resulting proof before it is published in its final form. Please note that during the production process errors may be discovered which could affect the content, and all legal disclaimers that apply to the journal pertain. 


\title{
The effect of wall slip on the stability of the Rayleigh-Bénard Poiseuille flow of viscoplastic fluids
}

\author{
Christel Métivier ${ }^{\mathrm{a}, *}$, Albert Magnin ${ }^{\mathrm{a}}$ \\ ${ }^{a}$ Laboratoire de Rhéologie, UMR 5520 (Université Joseph Fourier, CNRS, Grenoble INP), \\ Domaine universitaire, BP 53, 38041 Grenoble Cedex, France
}

\begin{abstract}
This work investigates the effect of wall slip on the stability of the Bingham Rayleigh-Bénard Poiseuille flow. The steady state of the Bingham plane Poiseuille flow is characterized by an unyielded region of $2 y_{b}$ width and two sheared regions close to the walls with both no-slip and slip conditions at the walls. A linear stability analysis of this flow with slip conditions is proposed in this paper. The slip boundary conditions case leads to flow destabilization compared with the results obtained in the no-slip case. Critical conditions are modified by varying $C_{f}$, the friction number. For $C_{f}<O(1)$, critical Rayleigh values $R a_{c}$ tend to that obtained with a free-free case. For $10<C_{f}<30, R a_{c}$ values decrease and reach a minimum in this zone. The value of $C_{f}$, for which $R a_{c}$ is minimal, varies slowly with the Bingham number $B$. For $C_{f}>30$ the flow is stabilized, i.e. $R a_{c}$ values increase and finally tend to that of the no-slip case when $C_{f}>1000$. Furthermore, for $1<C_{f}<10^{4}$, asymmetric modes were obtained. They are due to the slip boundary conditions at the walls.
\end{abstract}

Keywords: yield stress fluid, wall slip, stability analysis, thermo-convection

\section{Introduction}

Slip occurs in flows of concentrated dispersions due to the displacement of the disperse phase away from solid walls. Slip effects are usually observed when the disperse phase presents multi-micron sized particles or droplets (emulsion) or has a high molecular weight (polymers). When sheared with smooth surfaces, the concentrated dispersions exhibit apparent motion. Reviews on this topic have been proposed by Oldroyd [1] and Barnes [2]. Due to its practical interest, the slip of non-Newtonian fluids is widely studied. Numerous papers exist concerning observations of the slip effect in the case of concentrated dispersions. In particular, the slip of polymer microgels in rheometers has been

\footnotetext{
* Corresponding author

Email address: Christel.Metivier@ujf-grenoble.fr (Christel Métivier)
} 
studied for a long time (e.g. [3]-[5]). Furthermore, recent papers [6], [7] propose observations and measurements of slip in pastes of soft particles as well as elastohydrodynamical lubricating models of slip taking into account the physicochemical nature of the particle-wall interactions. It is shown that the attractive paste-wall interactions exhibit no wall slip and a bulk yield stress, while the repulsive interactions exhibit slip velocities and low slip yield stress.

Pearson and Petrie [8] first introduced a slip boundary condition giving a relationship between the slip velocity at the wall and the wall shear stress. Fortin et al. [9] used this model in the following form:

$$
\begin{aligned}
\widehat{\tau}_{\boldsymbol{t}}=-\widehat{c}_{f} \widehat{\boldsymbol{U}}_{\boldsymbol{t}}-\widehat{s}_{0} \frac{\widehat{\boldsymbol{U}}_{\boldsymbol{t}}}{\left|\widehat{\boldsymbol{U}}_{\boldsymbol{t}}\right|} & \text { iff } \quad\left|\widehat{\boldsymbol{U}}_{\boldsymbol{t}}\right| \neq \mathbf{0}, \\
\left|\widehat{\tau}_{\boldsymbol{t}}\right|<\widehat{s}_{0} & \text { iff }\left|\widehat{\boldsymbol{U}}_{\boldsymbol{t}}\right|=\mathbf{0},
\end{aligned}
$$

where the index $t$ means tangential component; i.e. $\widehat{\boldsymbol{U}}_{\boldsymbol{t}}=\widehat{\boldsymbol{U}}-(\widehat{\boldsymbol{U}} \cdot \boldsymbol{n}) \boldsymbol{n}$ and $\widehat{\boldsymbol{\tau}}_{\boldsymbol{t}}=\widehat{\boldsymbol{\sigma}} \cdot \boldsymbol{n}-[(\widehat{\boldsymbol{\sigma}} \cdot \boldsymbol{n}) \cdot \boldsymbol{n}] \boldsymbol{n}, \boldsymbol{n}$ being the unit outward vector. The hat notation is used for all dimensional variables. $\widehat{c_{f}}$ and $\widehat{s}_{0}$ represent respectively the friction coefficient and the slip yield stress. Experiments show that slip occurs when $\left|\widehat{\tau}_{\boldsymbol{t}}\right|>\widehat{s}_{0}$, otherwise no-slip conditions are observed. $\widehat{s}_{0}$ is found to be smaller than the bulk yield stress $\widehat{\tau}_{y}$. The classical linear slip condition is found when $\widehat{s}_{0}=0$. The analogy between Eq. (1) and the constitutive law of a Bingham fluid can be observed. Indeed, for $\widehat{\tau} \geq \widehat{\tau}_{y}$ the Bingham model reads:

$$
\widehat{\boldsymbol{\tau}}=\left(\frac{\widehat{\tau}_{y}}{\dot{\hat{\gamma}}}+\widehat{\mu}_{p}\right) \dot{\hat{\gamma}}
$$

The effect of wall slip on the flow of viscoplastic fluids was studied, for instance, by [10] who considered the Poiseuille flow in a square pipe. By means of an adaptive finite-element method, the different flow regimes are identified when the slip yield dimensionless number $S$, the friction number $C_{f}$ and the yield stress number $B$ vary.

To our knowledge, the influence of slip conditions on the stability of flows involving viscoplastic fluids has never been studied. Only a few studies e.g. [11]-[15] have been performed for Newtonian fluids. This is not surprising, since Newtonian liquids slip in very few situations (e.g. small scale flow configurations or hydrophobic solid surfaces). Considering a Newtonian plane Poiseuille flow with Navier slip conditions at the walls, papers [11], [12], [13] present linear stability analyses and show that wall slip stabilizes the flow. In this configuration, the transition to turbulence is delayed due to slip. However, [14] and [15] deal with the effect of slip boundaries on $2 \mathrm{D}$ thermal convection. They show that the flow is destabilized when the slip increases at the walls.

The aim of the present paper is to investigate the effect of slip near solid surfaces on the instability conditions when a viscoplastic fluid is involved. In particular, we propose to study the Rayleigh-Bénard Poiseuille (RBP) flow of a Bingham fluid. 
The instability of the Bingham RBP flow is considered in the no-slip case in [16], [17]. In these studies, the effect of the yield stress on critical conditions and on the evolution of the amplitude perturbation is investigated using linear and weakly non linear stability analyses respectively. Here, we propose to extend the study of [16] by considering slip conditions at the walls. In this respect, a linear stability analysis is developed considering dominant buoyancy forces, i.e. small values of the Reynolds number $R e$.

In section 2, all the governing equations are presented including the development of the steady state solution (section 2.1) and the perturbation set of linearized equations (section 2.2). The third section presents the numerical results as well as a discussion. The effect of the dimensionless numbers $S$ and $C_{f}$, is investigated and the results are compared with the no-slip configuration. The paper ends with a concluding section.

\section{Governing equations}

We consider the Poiseuille flow under an imposed axial pressure gradient of a yield stress fluid in a horizontal plane channel. The upper and lower walls are at constant temperatures, $\widehat{T}_{0}-\delta \widehat{T} / 2$ and $\widehat{T}_{0}+\delta \widehat{T} / 2$, respectively.

The dimensionless problem is obtained using the width $\widehat{L}$ of the plane channel as length-scale, and the thermal diffusion time $\widehat{L}^{2} / \widehat{a}$ between the two walls as the timescale; here $\widehat{a}$ is the thermal diffusivity of the fluid. In order to compare the slip conditions with the no-slip case, the maximum velocity $\widehat{U}_{0}$ of the noslip boundary conditions is used as the velocity scale. The stress and pressure scale is thus $\widehat{\mu}_{0} \widehat{U}_{0} / \widehat{L}$, with $\widehat{\mu}_{0}$ being the plastic viscosity. With the Boussinesq approximation, the governing equations read:

$$
\begin{gathered}
\boldsymbol{\nabla} \cdot \boldsymbol{U}=0, \\
\operatorname{Re} \boldsymbol{U}_{t}+\operatorname{Re} e^{2} \operatorname{Pr}(\boldsymbol{U} \cdot \boldsymbol{\nabla}) \boldsymbol{U}=\operatorname{Re} \operatorname{Pr}(-\nabla P+\nabla \cdot \boldsymbol{\tau})+\operatorname{Ra} T \boldsymbol{e}_{\boldsymbol{y}}, \\
T_{t}+\operatorname{Re} \operatorname{Pr}(\boldsymbol{U} \cdot \boldsymbol{\nabla}) T=\nabla^{2} T,
\end{gathered}
$$

where, $\boldsymbol{U}$ corresponds to the velocity vector, $T$ is the reduced temperature scaled with $\delta \widehat{T}, P$ the modified pressure, and $\boldsymbol{\tau}$ the deviatoric stress tensor. The velocity vector $\boldsymbol{U}$ is of the form $\boldsymbol{U}=U \boldsymbol{e}_{\mathrm{x}}+V \boldsymbol{e}_{\mathrm{y}}+W \boldsymbol{e}_{\mathrm{z}}$, where $U, V$ and $W$ are the velocity components and $\mathbf{e}_{\mathrm{x}}, \mathbf{e}_{\mathrm{y}}, \mathbf{e}_{\mathrm{z}}$ are unit vectors in the streamwise, transverse and spanwise directions respectively.

The dimensionless numbers are the Reynolds, $R e=\frac{\widehat{\rho} \widehat{U}_{0} \widehat{L}}{\widehat{\mu}_{0}}$, Prandtl, $\operatorname{Pr}=$ $\frac{\widehat{\mu}_{0}}{\widehat{\rho} \widehat{a}}$ and Rayleigh, $R a=\frac{\widehat{\rho} \alpha_{0} \widehat{g} \delta \widehat{T} \widehat{L}^{3}}{\widehat{\mu}_{0} \widehat{a}}$ numbers, $\widehat{\rho}$ is the fluid density, $\widehat{\alpha_{0}}$ is the thermal expansion coefficient and $\widehat{g}$ the gravitational acceleration.

As a remark, the scales chosen in this study are similar to those of the noslip walls case. In this sense, the effect of the boundary slip could be directly compared with the results shown in [17]. 
The constitutive law for a Bingham fluid is given by:

$$
\begin{array}{ll}
\tau=\mu \dot{\gamma} & \text { iff } \tau>B, \\
\dot{\gamma}=0 & \text { iff } \tau \leq B,
\end{array}
$$

with $\mu=1+\frac{B}{\dot{\gamma}}$ the effective viscosity and $B=\widehat{\tau}_{0} \widehat{L} /\left(\widehat{\mu}_{0} \widehat{U}_{0}\right)$ the Bingham number. The rate of strain tensor is denoted by $\dot{\gamma} ; \dot{\gamma}$ and $\tau$ are the second invariants of the rate of strain and deviatoric stress tensors, respectively.

Set (4) is completed by the boundary conditions at the walls with constant temperatures:

$$
T(-1 / 2)=T_{1}=1 / 2 \quad \text { and } \quad T(1 / 2)=T_{2}=-1 / 2,
$$

the wall slip is described as follows:

$$
\begin{array}{lll}
\boldsymbol{U}_{\boldsymbol{t}}( \pm 1 / 2)=-\frac{\boldsymbol{\tau}_{\boldsymbol{t}}( \pm 1 / 2)}{C_{f}}\left[1-\frac{S}{\left|\boldsymbol{\tau}_{\boldsymbol{t}}( \pm 1 / 2)\right|}\right] & \text { iff }\left|\boldsymbol{\tau}_{\boldsymbol{t}}( \pm 1 / 2)\right|>S, \\
\boldsymbol{U}_{\boldsymbol{t}}( \pm 1 / 2)=0 & \text { iff }\left|\boldsymbol{\tau}_{\boldsymbol{t}}( \pm 1 / 2)\right| \leq S,
\end{array}
$$

where the notation $|\cdot|$ represents the vector norm $|v|=(v \cdot v)^{1 / 2}$. In the twodimensional case, these conditions read:

$$
\begin{array}{lll}
U( \pm 1 / 2)=-\frac{\tau_{x y}( \pm 1 / 2)}{C_{f}}\left[1-\frac{S}{\left|\tau_{x y}( \pm 1 / 2)\right|}\right] & \text { iff }\left|\tau_{x y}( \pm 1 / 2)\right|>S \\
U( \pm 1 / 2)=0 & \text { iff }\left|\tau_{x y}( \pm 1 / 2)\right| \leq S
\end{array}
$$

The slip yield number $S$ and the friction number $C_{f}$ are defined respectively by:

$$
S=\frac{\widehat{s}_{0} \widehat{L}}{\widehat{\mu}_{0} \widehat{U}_{0}} \quad \text { and } \quad C_{f}=\frac{\widehat{c}_{f} \widehat{L}}{\widehat{\mu}_{0}},
$$

Finally, the boundary condition expressing the fact that the fluid does not cross the walls is given by:

$$
\boldsymbol{U} \cdot \boldsymbol{n}=0
$$

\subsection{Fully developed flow}

The steady state flow leads to a basic conductive state with a linear temperature profile given as follows

$$
T_{b}=-y
$$

The pressure is given by:

$$
P_{b}(x, y)=P_{r e f}-\frac{y^{2}}{2} \frac{R a}{R e P r}-\frac{2}{\left(1 / 2-y_{b}\right)^{2}} x,
$$

where $P_{\text {ref }}$ corresponds to a reference pressure. The stress field is described by the only non zero component $\tau_{x y}$ :

$$
\tau_{b x y}=\frac{\partial p}{\partial x} y=-\frac{2}{\left(1 / 2-y_{b}\right)^{2}} y
$$


and the velocity $\boldsymbol{U}_{b}=\left(U_{b}(y), 0,0\right)$ reads:

$$
U_{b}(y)= \begin{cases}1+\frac{1}{C_{f}}\left(\frac{B}{2 y_{b}}+S\right) & \text { for } \quad|y| \leq y_{b} \\ 1-\left(\frac{|y|-y_{b}}{1 / 2-y_{b}}\right)^{2}+\frac{1}{C_{f}}\left(\frac{B}{2 y_{b}}+S\right) & \text { for } \quad y_{b}<|y|<1 / 2\end{cases}
$$

where $2 y_{b}$ is the width of the central unyielded region as shown in Fig. 1. In Equation (16), it can be seen that $U_{s}=\frac{1}{C_{f}}\left(\frac{B}{2 y_{b}}+S\right)$ corresponds to the wall slip velocity. $U_{s}$ varies non-linearly with $C_{f}$ and $S$ as shown in Eq. (16) as well as in Fig. 1 which shows velocity profiles for different values of $C_{f}$ and $S$. The decrease in the friction coefficient as well as the increase in the value of $S$ lead to an increase in the slip velocity value $U_{s}$. The no-slip case is recovered for $U_{s}=0$, i.e. $C_{f} \rightarrow \infty$ and $S=0$. The perfect slip case, i.e. free of stress at the walls (free-free case) is obtained for $C_{f} \rightarrow 0$.

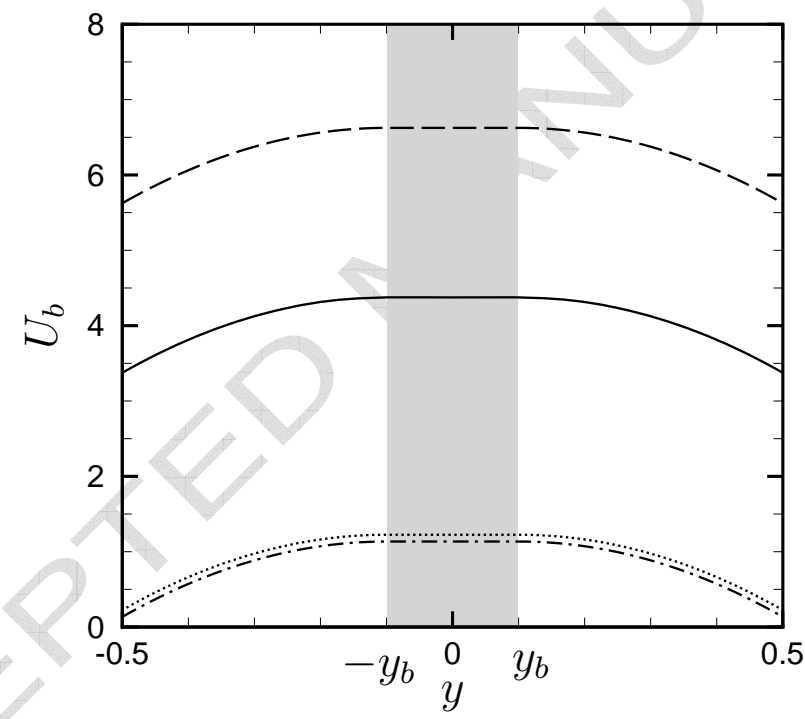

Figure 1: Velocity profiles for $y_{b}=0.1(B=1.25)$ and different values of $C_{f}$ and $S$. The gray zone represents the unyielded region. Dotted lines: $S=5, C_{f}=50$, dashed lines: $S=5, C_{f}=2$, dash-dotted lines: $S=0.5, C_{f}=50$, solid lines: $S=0.5, C_{f}=2$.

In addition, the yield surfaces position depends on the Bingham number $B$ via the following relation:

$$
B=\frac{2 y_{b}}{\left(y_{b}-1 / 2\right)^{2}} .
$$

Finally, comparing the two cases, i.e. no-slip boundary conditions and slip boundary conditions, the only difference is the value of the mean velocity which is increased by $U_{s}$ when slip occurs at walls. One can notice that, with $B$ 
constant, the shear rate and stress fields are not modified in either case. In particular, the wall stress value is $\tau_{w}=B /\left(2 y_{b}\right)>B$. As a consequence, the viscosity profile is also similar in the yielded regions.

\subsection{Linear stability analysis}

A small perturbation $A\left(\psi, \Theta, p, \pm y_{i}^{ \pm}\right)$is introduced into the fully developed flow. Here, $A$ corresponds to the amplitude of the perturbation and $\psi$ is the stream function, defined by $u=\partial_{y} \psi$ and $v=-\partial_{x} \psi$. The perturbation field is sought in the following form:

$$
\left(\psi, \pm y_{i}^{ \pm}, p, \Theta\right)=\left(f(y), \pm y_{1}^{ \pm}, p(y), \theta(y)\right) e^{\mathrm{i} \alpha(x-c t)},
$$

where $\alpha$ denotes the streamwise wave number and $\alpha c_{i}=\operatorname{Im}(\omega)$ denotes the wave speed.

Considering Eq. (18), the linearized equations of the perturbations (vorticity and energy equations) represent a generalized eigenvalue problem, with $c$ being the eigenvalue.

In the two yielded regions, the eigenvalue problem is written as:

$$
\begin{gathered}
\mathcal{L}_{1} f+\mathcal{L}_{2} \theta=c \mathcal{L}_{3} f \\
\mathcal{L}_{4} \theta+f=c \theta
\end{gathered}
$$

with $\mathcal{L}_{1}, \mathcal{L}_{2}, \mathcal{L}_{3}$ and $\mathcal{L}_{4}$, the following operators:

$$
\begin{aligned}
\mathcal{L}_{1} \equiv & \operatorname{Pr} \operatorname{Re}\left[U_{b}\left(D^{2}-\alpha^{2}\right)-D^{2} U_{b}\right] \\
& +\frac{\operatorname{Pri}}{\alpha}\left(D^{2}-\alpha^{2}\right)^{2}-4 \mathrm{i} \alpha \operatorname{Pr} B D\left[\frac{D}{\left|D U_{b}\right|}\right], \\
\mathcal{L}_{2} \equiv & \operatorname{Pr} \operatorname{Ra}, \\
\mathcal{L}_{3} \equiv & D^{2}-\alpha^{2}, \\
\mathcal{L}_{4} \equiv & \operatorname{Pr} \operatorname{Re} U_{b}-i \alpha+\frac{i}{\alpha} D^{2},
\end{aligned}
$$

where $D \equiv \partial_{y}$.

In the unyielded region, by combining the translation of the plug zone, in particular one writes $\partial_{x} \boldsymbol{u}\left(x, y_{i}^{ \pm}, t\right)=\mathbf{0}$, and the normal mode assumption leads to $f=u=v=0$. In this zone, the eigenvalue problem is reduced to:

$$
\begin{gathered}
f=0, \\
\mathcal{L}_{4} \theta=c \theta .
\end{gathered}
$$

At the yield surfaces $\left(y=y_{i}^{ \pm}\right)$, the yield conditions lead to:

$$
f\left( \pm y_{b}\right)=D f\left( \pm y_{b}\right)=0
$$

and

$$
D^{2} f\left( \pm y_{b}\right)= \pm y_{1}^{ \pm} D^{2} U_{b}\left( \pm y_{b}\right) .
$$

Actually, this last equation gives a condition for $y_{1}$, not for $f$. 
At the walls $(y= \pm 1 / 2)$, the constant temperature conditions read:

$$
\theta( \pm 1 / 2)=0
$$

At first order, the slip conditions, $u( \pm 1 / 2)=-\frac{\tau_{x y}^{\prime}( \pm 1 / 2)}{C_{f}}$, can also be written as follows:

$$
\left[D^{2} f+C_{f} D f-\alpha^{2} f\right]_{ \pm 1 / 2}=0 .
$$

In addition, the fact that the fluid does not cross the walls reads:

$$
v( \pm 1 / 2)=-f( \pm 1 / 2)=0 .
$$

Remarks:

(i) The no-slip case involves the same perturbation equations and boundary conditions except condition (25). Indeed, in the no-slip case, one obtains $f( \pm 1 / 2)=D f( \pm 1 / 2)=0$.

(ii) In the slip case, the asymptotic conditions lead to the following boundary conditions at the walls:

- For $C_{f} \rightarrow 0$

$$
f( \pm 1 / 2)=D^{2} f( \pm 1 / 2)=0 .
$$

These conditions are similar to the perfect slip conditions (free-free case).

- For $C_{f} \rightarrow \infty$

$$
f( \pm 1 / 2)=D f( \pm 1 / 2)=0
$$

These conditions are similar to no-slip boundary conditions.

\section{Results and discussion}

The set of equations (19)-(26) is solved numerically by means of a finite difference method. A second-order centered finite scheme is used in order to discretize the equations. The resulting problem represents an eigenvalue problem which is solved using the QZ algorithm implemented in Matlab 7.1. The numerical code was tested for the no-slip case, by comparing with the Newtonian case. The results converge for $N=201$ nodes, for which the Newtonian results $([18])$ are recovered with a discrepancy of $0.5 \%$. Considering the Bingham Rayleigh-Bénard Poiseuille flow with slip boundary conditions, the results converge for $N=301$.

A resulting spectrum of the slip configuration is presented in Fig. 2(a) for $S=2$ and $C_{f}=2$. The spectrum is plotted at criticality and is comparable to the one obtained in the no-slip configuration as shown in Fig. 2(b). The 


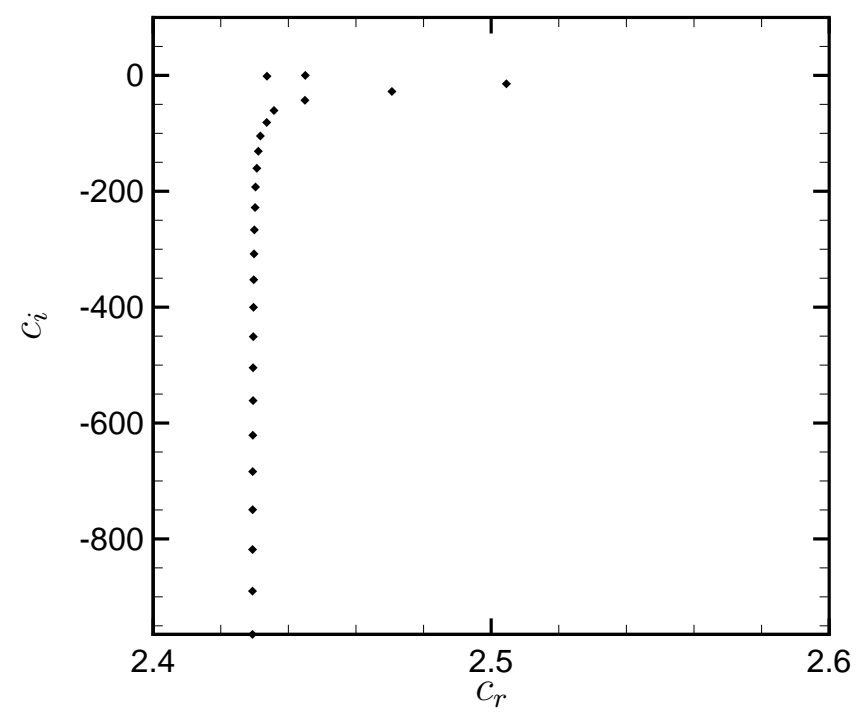

(a) Slip case: $S=2$ and $C_{f}=2$

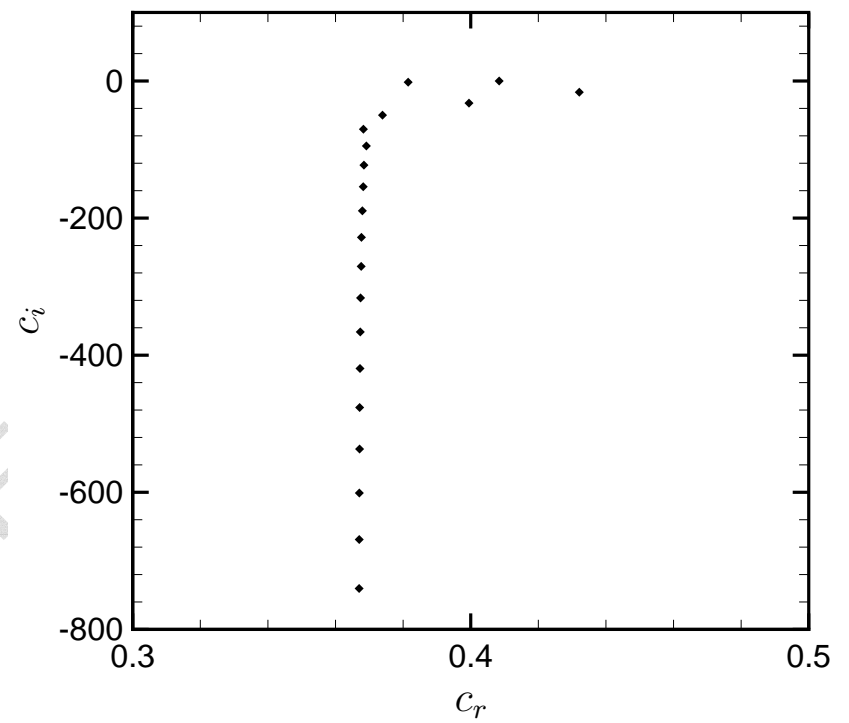

(b) No slip case

Figure 2: Spectrum of the largest eigenvalues for $\operatorname{Re}=0.01, \operatorname{Pr}=50, y_{b}=0.1(B=1.25)$ 
increase in the velocity phase can be observed, through the increase in $c_{r}$, in the slip case compared with the no slip case. This is due to the increase in the mean velocity of the basic flow.

Critical Rayleigh numbers are presented in Fig. 3 as a function of the Bingham number for $C_{f}=2$ and different values of $S$. In this figure, the no-slip case is represented by black squares and some symbols (circles and diamonds) corresponding to $S=5$ and $S=8$, since for low values of $B$ the Von Mises criterion leads to a no-slip condition, i.e. $\tau_{w} \leq S$ (Eq. (11)). The figure shows that slip conditions at the walls destabilize the flow decreasing the values of $R a_{c}$. With respect to $S=5$ and $S=8$, the variations in $R a_{c}$ are due only to stick-slip: either the fluid sticks at the walls $\left(\tau_{w} \leq S\right)$ with low $B$ or it slips $\left(\tau_{w}>S\right)$, since $\tau_{w}$ depends on $B$. In the both slip and no-slip cases, the Newtonian critical conditions are not recovered for $B \rightarrow 0$. This is due to the presence of the central plug zone in which the velocity perturbation vanishes (see [19] for further details). On the other hand, if only slip conditions are considered, one notices that variations in $S$ do not modify the values of the critical conditions since $R a_{c}$ remains constant by varying $S$, even in the limit case $S=0$. This is not surprising since the stress is weakly perturbed. In this sense the perturbed stress value remains close to that of the basic state, in particular at the walls $\tau_{x y}( \pm 1 / 2)=\tau_{w}>S$. Furthermore, $S$ does not have any influence on the stability analysis, i.e. Eqs (19)-(26).

The influence of the variations in $C_{f}$ is also investigated. Figure 4 represents critical conditions for different values of the friction number $C_{f}$ with $S=2$. The only cases considered with this value of $S$ are those in which the fluid slips at the walls, for all $B$. As said before, slip conditions at the walls destabilize the flow since the $R a_{c}$ values are smaller than that of the no-slip case (black squares). For $C_{f}>30$, critical conditions tend to that obtained in the no-slip case while for $C_{f}<10$, the $R a_{c}$ values tend to the perfect slip case. A transition region is observed for $10<C_{f}<30$. Actually, in this range of values, one observes that (i) if $B<B_{c}, B_{c}$ a critical value, then the $R a_{c}$ values tend to the no-slip conditions, (ii) if $B>B_{c}$, a large variation is observed in $R a_{c}$ and the flow is destabilized as shown in Fig. 4 (see circles and diamonds with dashed lines). Furthermore, it can be seen that $B_{c}$ varies slightly with $C_{f}$ as is also underlined in Fig. 5 (dashed lines). This figure, which represents $R a_{c}$ as a function of $C_{f}$ for different values of $B$, confirms that the variation in critical values, comparing the slip and the no-slip cases, is more significant in this transition region. With fixed values of $B$, one notices that $R a_{c}$ is constant when $C_{f}<O(1)$ and is equal to the value obtained for the limit case $C_{f} \rightarrow 0$, i.e. the free-free case. For $10<C_{f}<30$, the values of $R a_{c}$ decrease, reach a minimum at $C_{f m}=0(10)$ and then increase significantly. Finally, the $R a_{c}$ values tend to that of the noslip case with high values of $C_{f}\left(C_{f}>50\right)$. Figures 4 and 5 show clearly that the transition between the free-free and the no-slip cases is abrupt and depends on the values of $C_{f}$. In order to underline this dependency, calculations were performed, artificially setting $C_{f}=0$ only in the boundary conditions (25), i.e. for each value of $C_{f}$ the basic flow is retained but the perturbation boundary conditions at the walls are modified. If $C_{f}=0$ is imposed only in Eq. (25), 


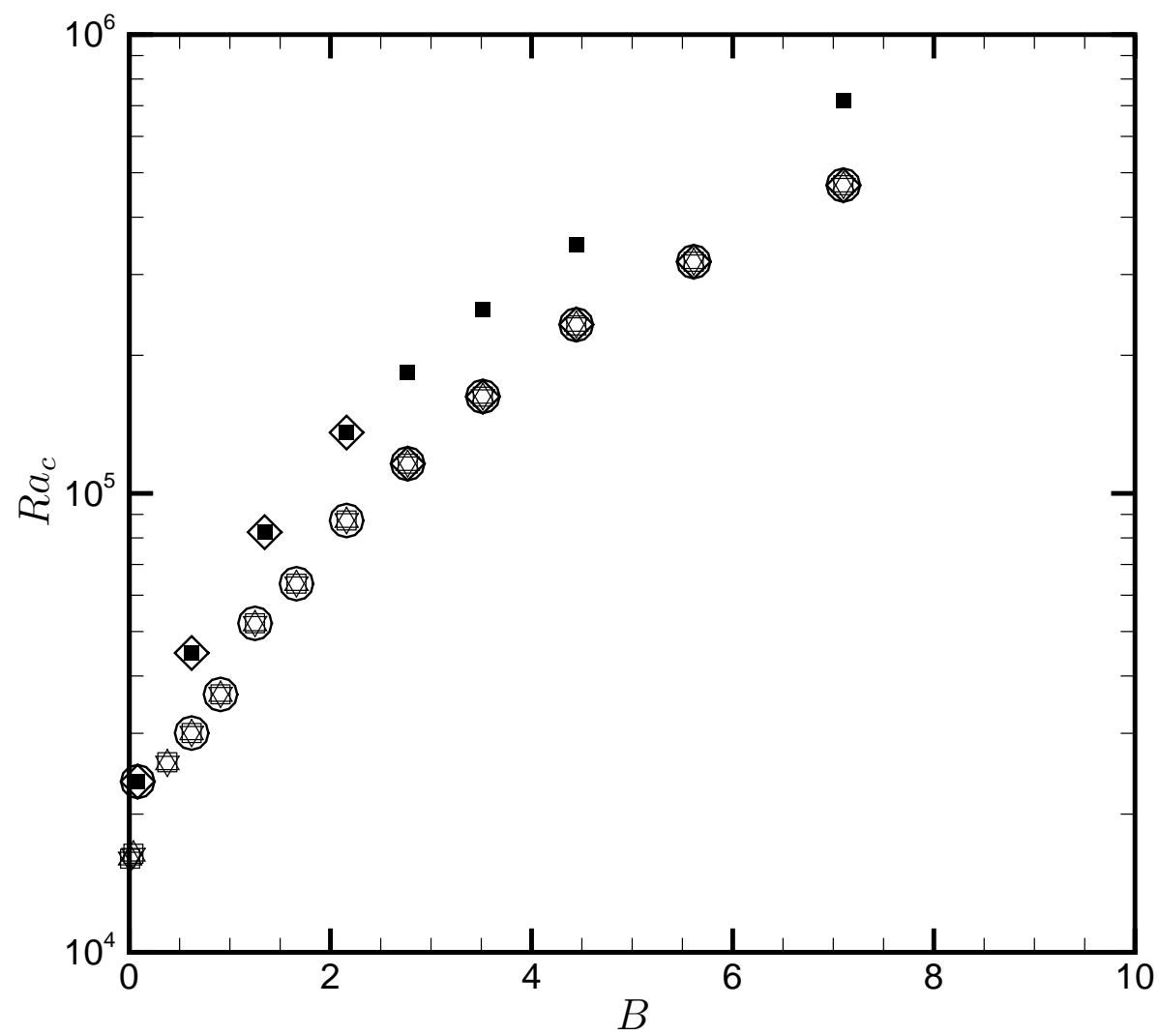

Figure 3: Critical Rayleigh number as a function of the Bingham number for different values of $S$ and $C_{f}=2, R e=0.01$ ( $\square$ : No-slip case, $\nabla: S=0, \square: S=0.5, \Delta: S=2, \circ: S=5, \diamond:$ $S=8)$

it can be seen that the critical conditions are constant (black squares in Fig. 5 ) for all tested values of $C_{f}$, and equal to that of the case $C_{f}=0.001$, i.e. $C_{f} \rightarrow 0$. The same goes for the resulting spectra and eigenmodes. This means that any variation in criticality (spectra, eigenmodes, critical conditions) is only due to the competition between the perfect slip case (free-free case Eq. (27)) and the no-slip case (Eq. (28)) via the value of $C_{f}$ in Eq. (25). Finally, the Bingham number has an influence on the variation in $R a_{c}$ since the difference, in the transition region, increases slightly with increasing $B$.

In a similar way to the no-slip case, the linear stability analysis leads to propagating convective patterns, on both sides of the unyielded region, in the form of traveling waves. The structure of the perturbed flow can be represented by the least stable mode in terms of temperature and stream function, as given by Figs. 6-9. In these figures, critical modes are represented as a function of $y$. 


\section{ACCEPTED MANUSCRIPT}

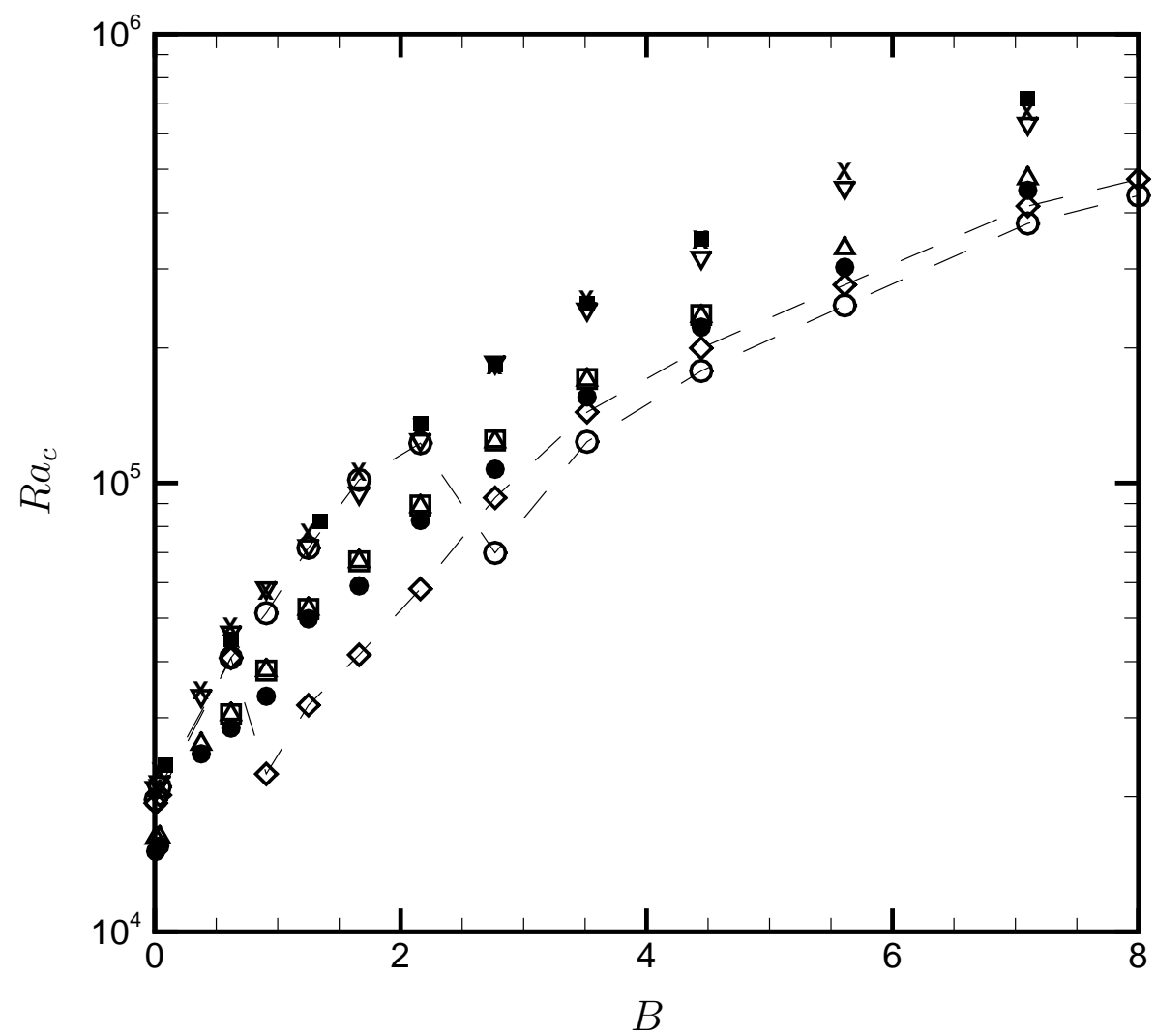

Figure 4: Critical Rayleigh number as a function of the Bingham number for different values of the friction number $C_{f}$ and $S=2, R e=0.01$ (ם: No-slip case, $\square: C_{f}=0.001, \Delta: C_{f}=0.01$, $\left.\bullet: C_{f}=5, \diamond: C_{f}=15, \circ: C_{f}=20, \nabla: C_{f}=50, \times: C_{f}=100\right)$.

In order to compare the modes $\boldsymbol{V}=(f, \theta)$, they are normalized as follows:

$$
\|\boldsymbol{V}\|=\frac{e^{\mathrm{i} x_{0}}}{\|\boldsymbol{V}\|_{\infty}} \boldsymbol{V}
$$

with $\|\boldsymbol{V}\|_{\infty}=\max _{y} \sqrt{\operatorname{Re}(V)^{2}+\operatorname{Im}(V)^{2}}$, the norm infinity, and $x_{0}=\operatorname{Arctan}\left(-\frac{\operatorname{Im}\left(\|\boldsymbol{V}\|_{\infty}\right)}{\operatorname{Re}\left(\|\boldsymbol{V}\|_{\infty}\right)}\right)$, the reference phase.

Figure 6 shows the critical modes obtained in the no-slip case. The modes are found to be symmetric and the maximum amplitude of the perturbation is attained by the real part of the stream function in the middle of each yielded region.

For low values of $C_{f}$, i.e. $C_{f}<0.1$, the critical modes are also symmetric, as shown in Fig. 7. However, a difference with the no-slip configuration can be 


\section{ACCEPTED MANUSCRIPT}

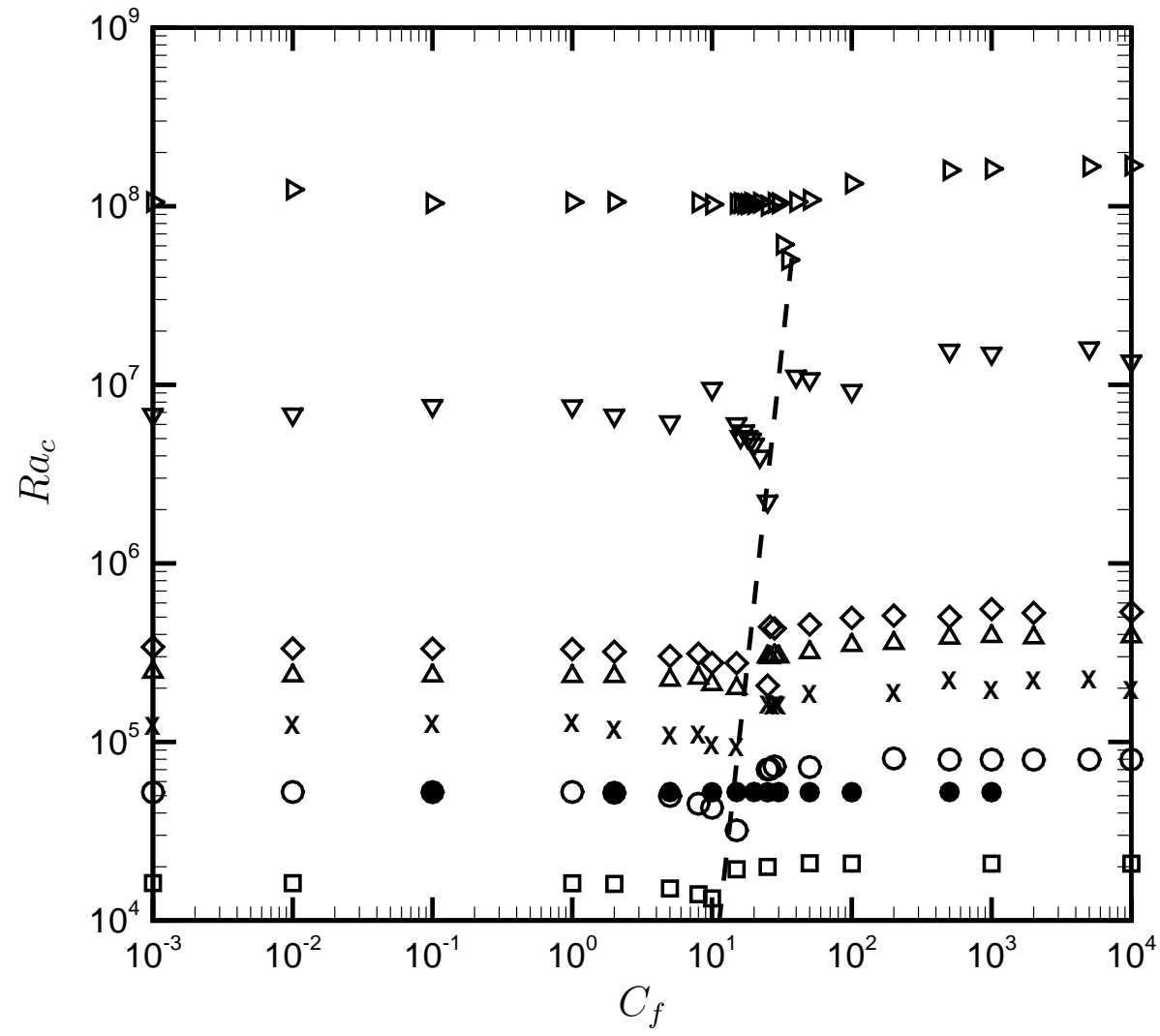

Figure 5: Critical Rayleigh number as a function of the friction number $C_{f}, S=2, R e=0.01$ (Squares: $y_{b}=0.001(B=0.008), \circ: y_{b}=0.1(B=1.25), \bullet: y_{b}=0.1$ and $C_{f}=0$ in Eq. $(25), \times: y_{b}=0.16(B=2.77), \Delta: y_{b}=0.2(B=4.44), \diamond: y_{b}=0.22(B=5.61), \nabla: y_{b}=0.34$ $(B=26.56), \triangleright: y_{b}=0.40(B=80)$. The dashed line indicates the minimal values of $R a_{c}$.

observed via the derivative of $f$ at the walls given by the boundary conditions (27) when $C_{f} \rightarrow 0$. Finally, the destabilization of the flow is due to perfect slip conditions in which no tangential stresses act. This is in agreement with the decrease in $R a_{c}$ in the Rayleigh-Bénard Newtonian case (see[20]) when the (no-slip) rigid surfaces case $\left(R a_{c}=1707.76\right)$ is compared with the free surfaces case $\left(R a_{c}=657.51\right)$.

Asymmetric modes can be observed for $1<C_{f}<10^{4}$, as represented in Figs. 8-10. The increase in the values of $C_{f}$ increases the differences between the extrema of the mode $f$ in the two yielded regions. On the other hand, this increasing asymmetric effect goes with a mean decrease in the intensity of the perturbation via $f$ and $\theta$. As said before, the asymmetry does not involve significant variations in $R a_{c}$, if the results obtained by varying $C_{f}$ from 1 to 10 are compared. 


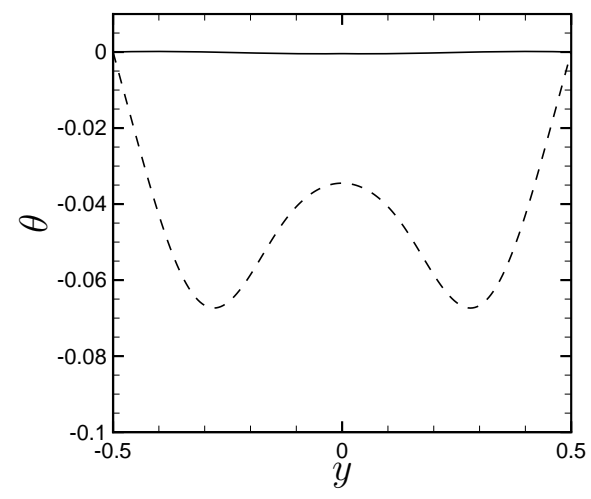

(a) Temperature

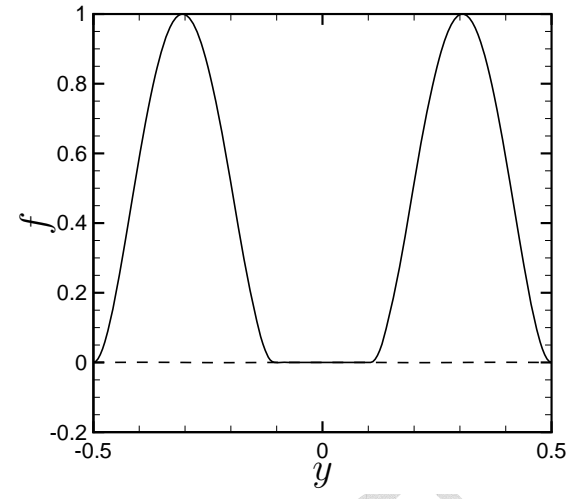

(b) Stream function

Figure 6: Critical modes for the no-slip case, $y_{b}=0.1(B=1.25), R e=0.01$ (solid line: real part, dashed line: imaginary part).

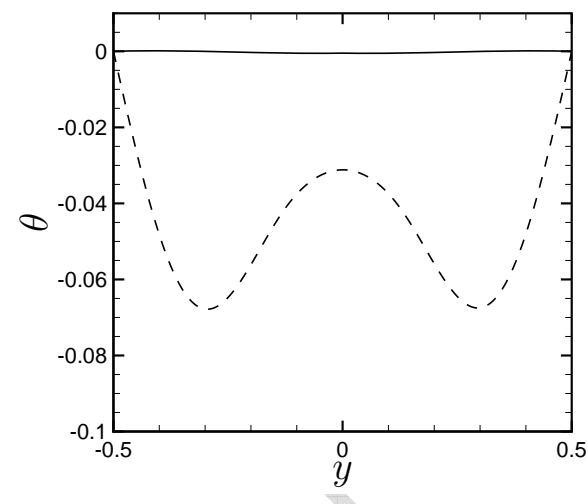

(a) Temperature

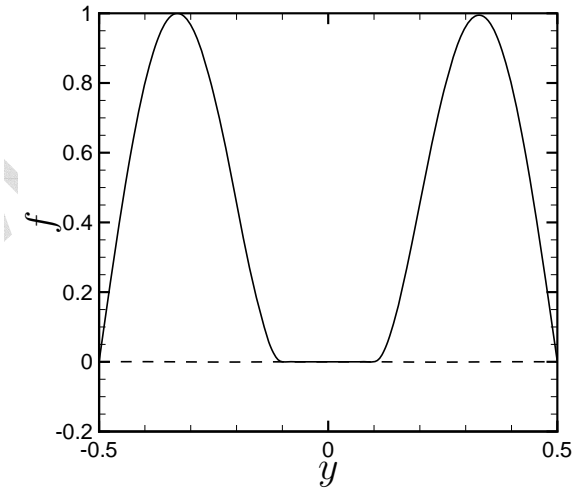

(b) Stream function

Figure 7: Critical modes for $C_{f}=0.01, S=2, y_{b}=0.1(B=1.25), R e=0.01$ (solid line: real part, dashed line: imaginary part).

Furthermore, with increasing $C_{f}$ values, the break in symmetry is still observed even when $10<C_{f}<10^{4}$ while the $R a_{c}$ values tend to those obtained in the no-slip configuration as shown in Fig. 9. Actually, the modes obtained in the no-slip configuration can be recovered with larger values of $C_{f}$, that is to say $C_{f}=O\left(10^{4}\right)$, as can be seen in Fig. 10. As expected, for $C_{f} \rightarrow \infty$, the no-slip case is recovered in terms of critical conditions as well as flow conditions, e.g. the perturbation modes.

Furthermore, the thermoconvective rolls are depicted via the isovalues of normal modes $\Psi$ and $\Theta$ in Fig. 11. Obviously, the asymmetry of the modes is 


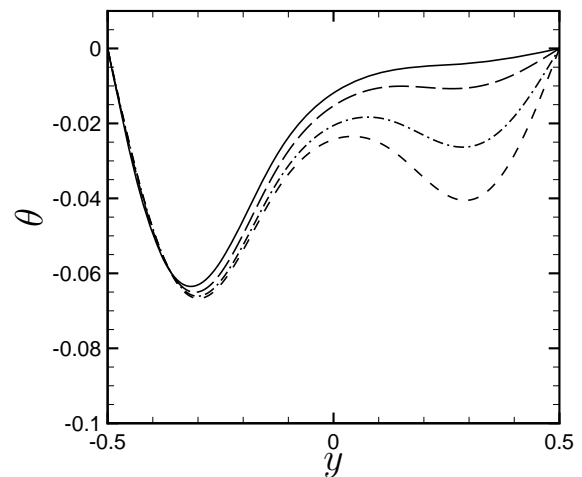

(a) Temperature - Imaginary part

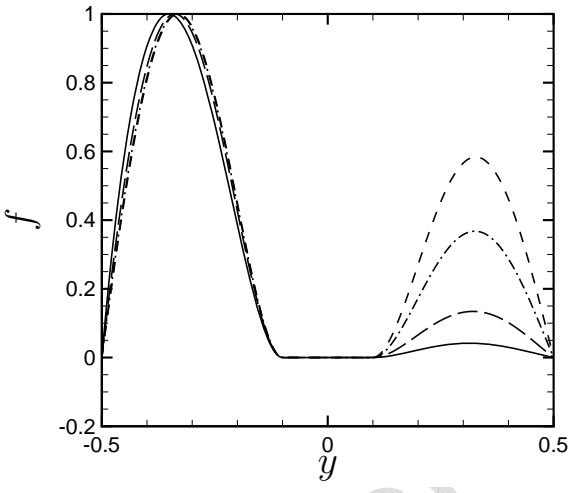

(b) Stream function - Real part

Figure 8: Critical modes for different values of $C_{f}, S=2, y_{b}=0.1(B=1.25), R e=0.01$ (solid line: $C_{f}=10$, long dashed line: $C_{f}=5$, dash-dotted line: $C_{f}=2$, dashed line: $\left.C_{f}=1\right)$.

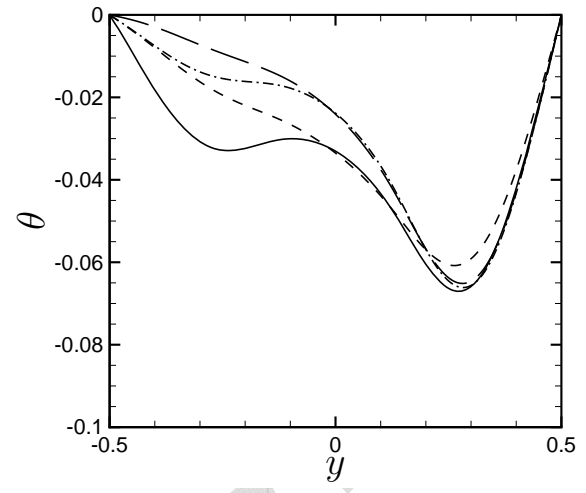

(a) Temperature - Imaginary part

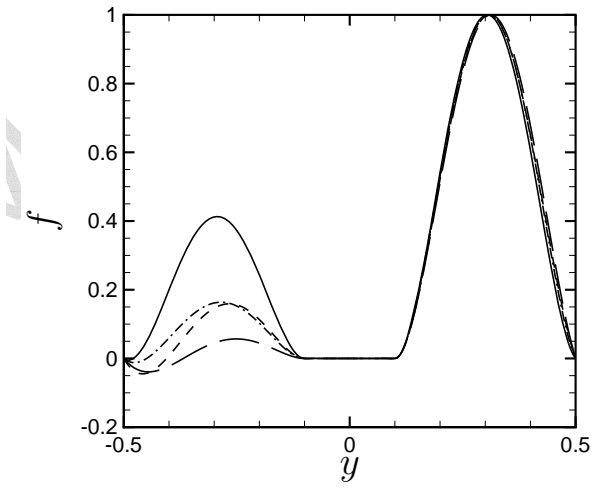

(b) Stream function - Real part

Figure 9: Critical modes for $S=2, y_{b}=0.1(B=1.25), R e=0.01$ (solid line: $C_{f}=100$, dash-dotted line: $C_{f}=50$, dashed line: $C_{f}=30$, long dashed line: $C_{f}=25$ ).

also observed via a decrease in the intensity of the perturbation in one yielded region: either the upper one or the lower one depending on whether $C_{f}$ values are low or large respectively.

The origin of the asymmetry in terms of perturbation modes is due only to $C_{f}$. Actually, as said before, when $C_{f}$ is artificially set to zero in boundary condition (25), the perturbation modes are symmetric and are similar to the ones obtained with $C_{f}=0.01$ (Fig. 7). The asymmetry of the modes is due only to the competition between the (derivatives at the walls of the) modes obtained in the no-slip case (Fig. 6) and the ones obtained in the free-free case 


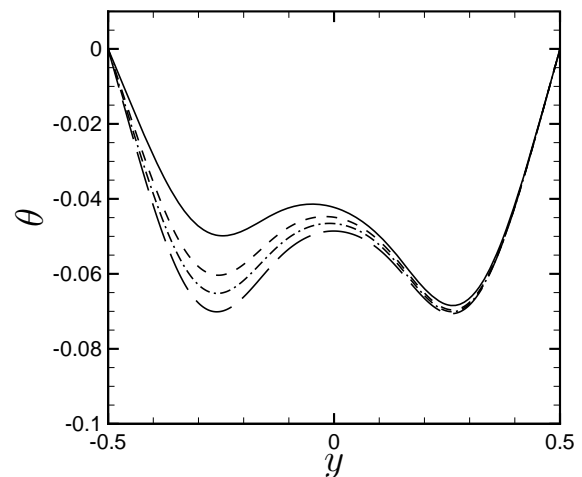

(a) Temperature - Imaginary part

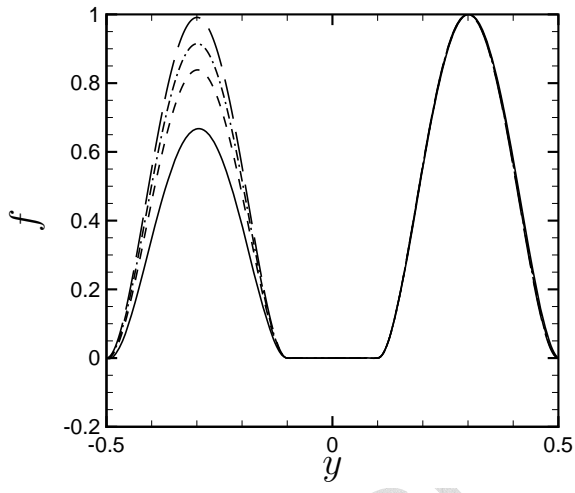

(b) Stream function - Real part

Figure 10: Critical modes for $S=2, y_{b}=0.1(B=1.25), R e=0.01$ (solid line: $C_{f}=200$, dashed line: $C_{f}=500$, dash-dotted line: $C_{f}=1000$, long-dashed line: $\left.C_{f}=10000\right)$.

(Fig. 7) via the value of $C_{f}$ in (25).

\section{Conclusion}

The linear stability of the plane Bingham Rayleigh-Bénard Poiseuille flow was studied considering slip conditions at the walls. The effect of the two dimensionless numbers $S$ and $C_{f}$, which characterize the wall slip, was investigated. The numerical results show that the wall slip leads to destabilize the flow since the critical Rayleigh values are smaller than the ones obtained in the no-slip case. In the range of values tested, one observes that variations in $S$ do not modify the critical conditions, while variations in $C_{f}$ do. We showed that (i) for $C_{f}<0(1)$, the critical conditions tend to the free-free case, (ii) for $C_{f}>30$, the critical Rayleigh values increase, (iii) the no-slip case is recovered in terms of critical conditions when $C_{f} \geq 100$. The effect of $C_{f}$ was also underlined via the perturbation modes. In a range of values such as $10<C_{f}<10^{4}$, asymmetric modes were observed in terms of stream function and temperature. This is clearly the consequence of the competition between the free-free case and the no-slip case through the slip conditions (25).

In conclusion, it is of great importance to pay attention to wall conditions, in particular in experiments, since critical conditions can be modified by considering either no-slip or slip conditions.

\section{Acknowledgments}

The authors' work was supported by the French National Research Agency (ANR), via the grant called "ThiM". The authors are also grateful to the Research Group AmeTH. 


\section{References}

[1] Oldroyd J.G, Non-Newtonian flow of solids and liquids, In Eirich F.R. (editor), Rheology: Theory and applications, Vol. 1, Academic Press Inc., New York (1956) 653-682.

[2] H.A. BARnes, A review of the slip (wall depletion) of polymers solutions, emulsions and particle suspensions in viscosimeters: it causes, character, and cure, J. Non-Newtonian Fluid Mech., 36 (1995) 85-108.

[3] G.V. Vinogradov \& G.B. Froisheter \& K.K. Trilisky, The flow of plastic disperse systems in the presence of the wall effect, Rheol. Acta, 14 (9) (1975) 765 .

[4] A. Magnin \& J.M. Piau, Cone-and-plate rheometry of yield stress fluids. Study of an aqueous gel, J. Non-Newtonian Fluid Mech., 56 (1990) 221-251.

[5] J.M. PIAU, Carbopol gels: Elastoviscoplastic and slippery glasses made of individual swollen sponges. Meso- and macroscopic properties, constitutive equations and scaling laws, J. Non-Newtonian Fluid Mech., 144 (2007) 1-29.

[6] S.P. Meeker \& R.T. Bonnecaze \& M. Cloitre, Slip and Flow in Soft Particles Pastes, Phys. Rev. Lett., 92 (19) (2004) 198302.

[7] J.R. Seth \& M. Cloitre \& R.T. Bonnecaze, Influence of short-range forces on wall-slip in microgel pastes, J. Rheol., 52 (5) (2008) 1241-1268.

[8] J.R.A. Pearson \& C.J.S. Petrie, Proceedings of the Fourth International Congress on Rheology, Part 3, Wiley, New-York, (1965), pp. 265282.

[9] A. Fortin \& D. Cté \& P.A. TAnguy, On the imposition of friction boundary conditions for the numerical simulation of Bingham fluid flows, Comput. Meth. Appl. Mech. Eng. 88 (1991) 97109.

[10] N. Roquet \& P. Saramito, An adaptative finite element method for viscoplastic flows in a square pipe with stick-slip at the wall, J. Non-Newtonian Fluid Mech., 155 (2008) 101-115.

[11] J.M. Gersting, Hydrodynamic stability of plane porous slip flow, Phys. Fluids, 17 (2126) (1974).

[12] E. Lauga \& C. Cossu, A note on the stability of slip channel flows, Phys. Fluids, 17 (088106) (2005).

[13] T. Min \& J. Kim, Effects of hydrophobic surface on stability and transition, Phys. Fluids, 17 (108106) (2005).

[14] L.S. Kuo \& P.H. Chen, Effects of slip boundary conditions on RayleighBénard convection, J. Mech., 25 (2009) 205-212. 
[15] L.S. Kuo \& W.P. Chou \& P.H. Chen, Effects of slip boundaries on thermal convection in 2D box using Lattice Boltzmann method, Int. J. Heat Mass Transfer, 52 (2011) 1340-1343.

[16] C. Métivier \& C. Nouar, On linear stability of Rayleigh-Bénard Poiseuille flow of viscoplastic fluids, Phys. Fluids 20 (104101) (2008).

[17] C. Métivier \& C. Nouar \& J.P. Brancher, Weakly nonlinear dynamics of thermoconvective instability involving viscoplastic fluids, J. Fluid Mech., 660 (2010) 316-353.

[18] X. Nicolas, Revue bibliographique sur les écoulements de PoiseuilleRayleigh-Bénard : écoulements de convection mixte en conduites rectangulaires horizontales chauffées par le bas, Int. J. Heat and Mass Transfer, 43 (2000) 589.

[19] C. MÉtivier \& C. Nouar \& J.P. Brancher, Linear stability involving the Bingham model when the yield stress approaches zero, Phys. Fluids 17 (104106) (2005).

[20] S. Chandrasekhar, Hydrodynamic and hydromagnetic stability, Oxford: Clarendon press, (1961). 

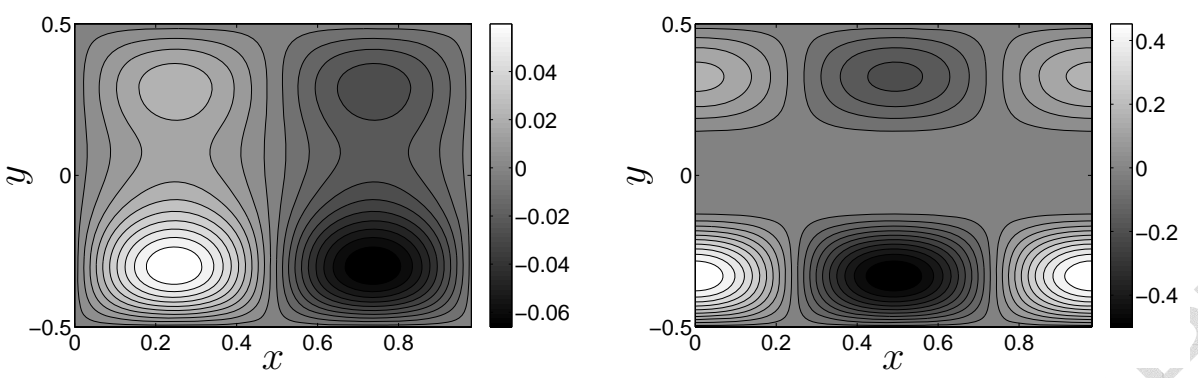

(a) $S=2 C_{f}=2$
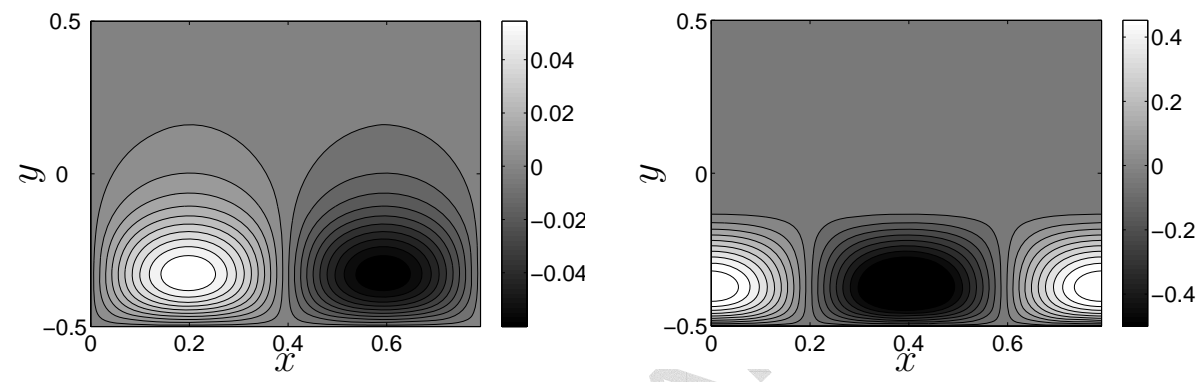

(b) $S=2 C_{f}=15$
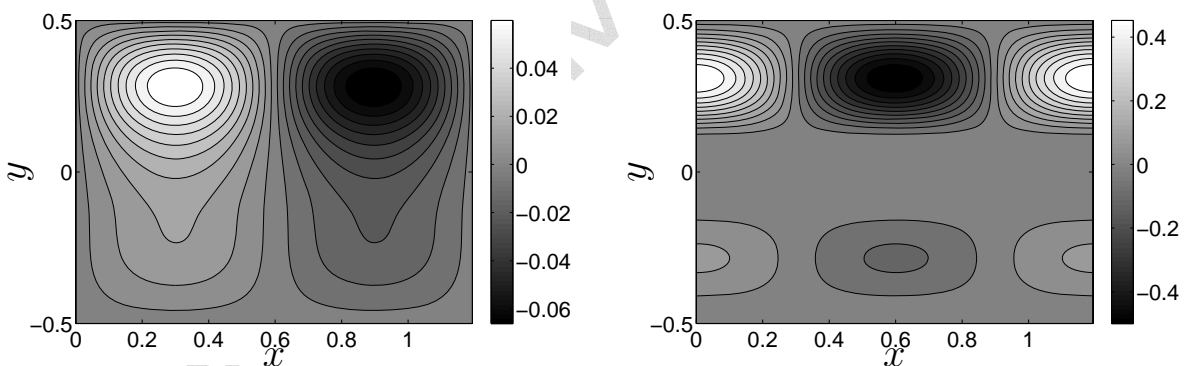

(c) $S=2 C_{f}=50$
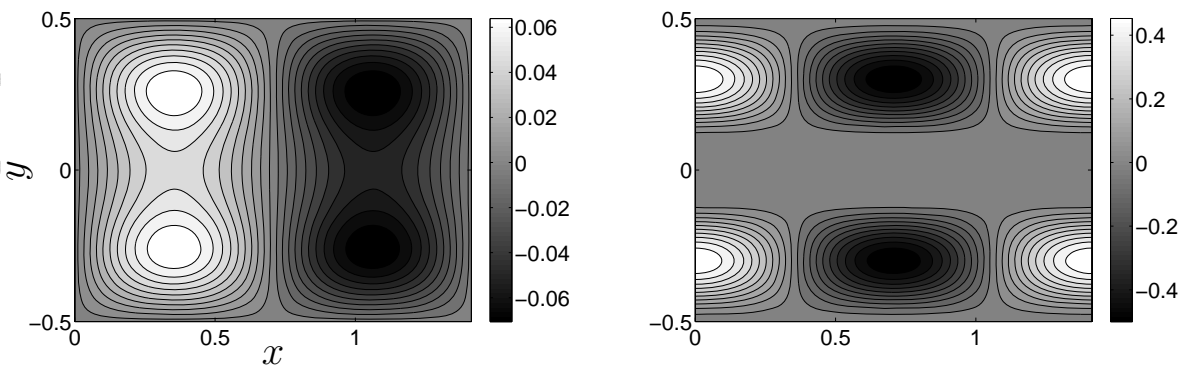

(d) $S=2 C_{f}=10000$

Figure 11: Isovalues of temperature (left) and stream function (right) perturbation for $y_{b}=0.1$ $(B=1.25), R e=0.01, P=50$ by varying $C_{f}$. 
The effect of wall slip on the stability of the Rayleigh-Bénard Poiseuille flow of viscoplastic fluids

\section{by Christel Métivier and Albert Magnin}

- Effect of wall slip on the linear stability of the Bingham Rayleigh-Bénard Poiseuille (RBP) flow.

- Slip boundary conditions destabilize the RBP flow compared with the no-slip case.

- Criticality depends on the Bingham number $B$ and on the friction number $C_{f}$.

- Asymmetric modes are obtained for $1<C_{f}<10^{4}$ 